\title{
Dream to explore: 5-HT2a as adaptive temperature parameter for sophisticated affective inference
}

\author{
Adam Safron $^{1}$ and Zahra Sheikhbahaee ${ }^{2}$ \\ ${ }^{1}$ Center for Psychedelic and Consciousness Research, Department of Psychiatry \& \\ Behavioral Sciences, Johns Hopkins University School of Medicine, Baltimore, MD \\ 21224, USA \\ ${ }^{2}$ David R. Cheriton School of Computer Science, University of Waterloo, ON, Canada \\ asafronegmail.com \\ zsheikhb@uwaterloo.ca
}

\begin{abstract}
Relative to other neuromodulators, serotonin (5-HT) has received far less attention in machine learning and active inference. We will review prior work interpreting 5-HT1a signaling as an uncertainty parameter with opponencyto dopamine. We will then discuss how 5-HT2a receptors may promote more exploratory policy selection by enhancing imaginative planning (as sophisticated affective inference). Finally, we will briefly comment on how qualitatively different effects may be observed across low and high levels of 5-HT2a signaling, where the latter may help agents to change self-adversarial policies and break free of maladaptive absorbing states in POMDPs.
\end{abstract}

Keywords: Serotonin, 5-HT1a, 5-HT2a, Sophisticated Active Inference, Affective Inference, Exploration, Exploitation, Imagination, Planning, Consciousness.

\section{Introduction}

Serotonin (5-HT) is a phylogenetically ancient monoamine neuromodulator found in all life forms, and which in mammals involves at least 14 distinct receptors that canbe subdivided into 7 sub-classes [1]. This divergence of 5-HT systems arose through a process of evolutionary divergence via gene duplication and subsequent specialization of receptor subtypes and associated pathways [2]. This diversity may seem to suggest limited utility for attempting to recapitulate 5-HT-related functions in artificial systems. However, we propose that a substantial portion of 5-HT-related functionality may be obtained by focusing on the 1a and 2 a receptors. We suggest this seemingly myopic focus on these two receptor classes may constitute a fruitful research direction on account of their highly conserved status in evolution, their relatively broad distribution in mammalian brains, as well as the common organismic significance of 5-HT signaling implicated by the mass-release of these diffusely acting neuromodulators from concentrated neurons in midbrain nuclei. While understanding the full diversity of 5HT signaling will likely be illuminating with respect to abilities to differentially modify 
various neural processes - with potentially common organismic significances-we believe a focus on 1a and $2 \mathrm{a}$ receptors will provide both maximal explanatory purchase and a foundation upon which subsequent modelling may proceed.

Cortex is highly populated by both 5-HT1a and 5-HT2a receptors [1]. 5-HT1a receptors have primarily inhibitory effects on neurons, and are functionally associated with somewhat subtle effects on mood, uncertainty, and the learning of complex behaviors, whereas 5-HT2a receptors have primarily excitatory effects and appear to have more pronounced effects on affect and cognitive processes [3]. 5-HT2a receptors also mediate the primary mechanism of action for hallucinogenic drugs such as lysergic acid diethylamide (LSD), psilocybin, and N,N-dimethyltryptamine (DMT) [4]. These compounds are widely known for inducing states of altered perception, thought, and feeling, with similarities to lucid dreaming; in this way, psychedelic states share features with both dreaming and waking consciousness [5].

The functionality of 5-HT1a and 5-HT2a receptors has respectively been associated with either passive or active coping strategies in the face of threat (or uncertainty) [6]. In this view, 5-HT1a signaling enables adaptive responses to mild-to-moderate stress through affective regulation and the inhibition of (disinhibitory) dopaminergic processes. 5-HT2a signaling, in contrast, is upregulated during more intense states of challenge - potentially including uncertainty with respect to highly valued goals - so allowing for both increased behavioral flexibility and neural plasticity [7].

The Free Energy Principle and Active Inference (FEP-AI) framework characterizes organisms as kinds of generative models [8], [9], with brains functioning as cybernetic control systems for embodied agents as they attempt to minimize uncertainty with respect to realizing their goals. These (both implicitly and explicitly) valued goals are understood as Bayesian prior preferences over likely outcomes that allow such systems to maintain their forms on evolutionary and developmental timescales. Towards this end, an FEP-AI agent maximizes model evidence for its existence by minimizing expected (variational) free energy (i.e., cumulative precision-weighted prediction errors) between its world model and sensory observations. This expected free energy minimization (and thereby self-model-evidence maximization) is realized either via perception (updating world models) or action (enactively updating world states). According to hierarchical predictive processing (HPP) models, cortical processes - and potentially biological systems more generally - continuously generate top-down predictions of bottom-up information at multiple levels of hierarchical abstraction. Notably, each level of these hierarchical generative models has varying levels of spatial and temporal granularity with respect to the latent system-world states it attempts to predict and alter through active inference, so allowing for multi-scale models with varying degrees of temporal depth and counterfactual richness [10]. In HPP, bottom-up observations are (efficiently) encoded as prediction errors, which ascend to higher cortical levels to update generative models when not predictively suppressed by topdown prior expectations. At the highest levels of abstraction in cortical hierarchies, maximal explanatory power may be found through models related to complex processes such as those underlying various forms of selfhood and self-consciousness [11]-[13].

Within FEP-AI, conscious planning is understood as "sophisticated active inference," in which agents generate imagined sequences of counterfactual outcomes 
through rolling out mental simulations of different patterns of action/policy selection [14], [15]. This imaginative planning takes the form of a deep tree search over counterfactual observations and actions, where different rollouts of simulated actions allow for exploring different branches of decision trees. This sophisticated active inference is governed by a singular objective function(al) of expected free energy, which achieves balance with respect to exploration-exploitation tradeoffs by selecting governing models that neither overfit nor underfit patterns of data in shaping perception and action. At perhaps the highest level of organization, patterns of action/policy selection via counterfactual processing (e.g., simulated movements through space) are orchestrated by the hippocampal/entorhinal system [16], [17], where these spatiotemporal trajectories may be understood as constituting the stream of consciousness [13].

Below we describe parts of our ongoing explorations of ways in which the functional significances of 5-HT1a and 5-HT2a receptors may be understood through the lens of FEP-AI. If accurate, these models may provide a unified account of the roles of 5-HT in adaptive behavior with implications for machine-learning, neuropsychology, and evolutionary-developmental biology. We will characterize ways in which both 5-HT1a and 5-HT2a receptors influence the degrees to which agents initiate imaginative planning and offline learning via mental simulations, as opposed to more immediately releasing policies for overt goal-seeking behaviors. Finally, we will address some potential misconceptions about the functionality of different levels of 5-HT2a signaling, and further establish potentially fruitful connections to meta-reinforcement learning.

\section{5-HT1a Receptors}

5-HT1a receptors are found in different layers of the cortex, but they are most strongly expressed in layers V and VI [1]. 5-HT1a receptors suppress pyramidal cell activity by increasing rectifying $\mathrm{K}^{+}$currents, and have also been found to inhibit gamma oscillations in the hippocampus [18], potentially indicating reduced sensitivity to overall organismic prediction error [19]. This is opposite to the effects for DA (and in some respects 5-HT2a) signaling, which in FEP-AI is understood as enhancing the precision of bottom-up prediction errors [20], [21], so promoting the sensitivity of behavioral response to rewards. 5-HT1a signaling, in contrast, would instead promote deliberation and patience with respect to policy deployment [22]-[24].

Substantial experimental evidence has demonstrated opponency between dopaminergic (DA) and serotoninergic (5-HT) signaling [25], [26], which appear to be respectively associated with situations characterized by either more appetitive or aversive states. For biological organisms, appetitive motivational systems encourage approach while aversive systems promote avoidance and withdrawal. However, this is not to say that serotonin creates aversion, but rather that 5-HT signaling tends to be enhanced for situations in which organisms experience stress and uncertainty with respect to their ability to achieve their goals [27], including the fundamental goal of 
survival. This is consistent with modulation of 5-HT1a neurons in the dorsal raphe nucleus [28], since release of action policies associated with either more passive or active coping ought to be modulated by the expected value of different patterns of enaction. However, firing rates for serotonergic also correlate with uncertainty more generally, indicating sensitivity to surprising events irrespective of the value of particular rewards. Opponency is observed in 5-HT1a and DA systems in terms of mutual inhibition of release and differential shaping of modulated systems [29]. However, their interactions can also produce synergy, both in terms of differentially parameterizing the nature of imaginative planning (e.g. with more or less confidence), and also in terms of providing a dynamic tension via their opponency, since pursuing complex goals require capacities for flexible adaption in response to environmental changes [30]-[33].

5-HT1a receptors are found as somatodendritic autoreceptors in raphe nuclei, as well as in postsynaptic sites in neocortex, hippocampus, and other "limbic" structures such as the amygdala and homeostatic regulatory nuclei of the hypothalamus [1]. Through inhibiting excitatory neurotransmission, 5-HT1a autoreceptors can both help with passively coping with stressful events by attenuating prediction error, and also help to promote more adaptive behavior by providing more time for planning in the face of uncertain circumstances. This inhibition of overt behavior affords both an opportunity for being informed by more complex world modeling, as well as an opportunity for adaptively calibrating world models by imbuing imaginative rollouts with greater uncertainty (i.e., implicitly functioning as a temperature parameter) [34]. Taken together, these functions allow for more flexible behavior via planning, as well as enhanced exploration and policy generalization via imagining the pursuit of goals under uncertain and potentially challenging conditions. Functionally speaking, this would be extremely sensible for a parameter that tends to be elevated in not just stressful circumstances, but also in situations involving the satiation of organismic drives such as eating, and possibly social contact [35]-[38]. That is, once goals are realized, a shift from exploration to exploitation would be both an adaptive foraging strategy and proximate mechanism for lifelong learning. Further, the more passive behavior encouraged by attenuation of action readiness would likely also be an adaptive response for an agent facing potential threats from circumstances that may overwhelm its present control abilities.

Thus, in FEP-AI terms, 5-HT1a signaling would be understood as promoting sophisticated inference via imaginative rollouts of predicted (or postdicted) patterns of enaction under conditions of reduced precision over counterfactually-deployed policies [14]. This would correspond to an agent experiencing relatively lower levels of confidence while entertaining counterfactual policies, but also with reduced "affective charge" [15], which one would normally expect to be stronger in a negative direction under conditions of reduced certainty with respect to realizing prior preferences. In these ways, 5-HT1a signaling would promote adaptive responses to challenging (and potentially novel) environments by increasing tolerance with respect to uncertainty through stress moderation, so allowing for more flexible and sophisticated forms of cognition and behavior [22]-[24]. 
The effects of different levels of 5-HT1a signaling may have profound functional consequences. For example, the ascending serotonergic pathway from the dorsal raphe nucleus and its effects on the amygdala and frontal cortex may promote adaptive reshaping conditioned fear responses [39], [40]. The basolateral nucleus of the amygdala contributes to behavioral changes in the face of emotional events and associated stimuli, including in response to stressors such as social defeat and other fearful circumstances [41], [42]. However, activation of 5-HT1a postsynaptic receptors in the dorsal hippocampus and amygdala facilitate extinction of fear-conditioned behaviors, consistent with the proposed roles of serotonin in facilitating coping in the face of threat [39]. With respect to potentially synergistic interactions between neuromodulatory systems, DA may be understood as providing a learning rate signal that influences the degree to which an agent updates its predictions in response to novel experiences [43]. This learning rate would influence the degree to which reward prediction errors shape policy selection, which if excessive could result in impulsivity by having presently estimated rewards promote more reactive forms of policy selection [44]. 5-HT1a, in contrast, would promote modeling with greater temporal depth and counterfactual richness, providing opportunities for meta-learning with sensitivity to (and ability to adaptively cope with) uncertain circumstances [24]. Notably, with respect to pathological states such as the rumination associated with depression and the impulsive aggression associated with antisocial behavior, low 5-HT1a in the medial prefrontal cortex appears to be associated with more perseverative tendencies and reduced abilities to adapt to novel environments [45], [46].

\section{$3 \quad$ 5-HT2a Receptors}

5-HT2a receptors are most strongly expressed in high-level association cortices [1], including the "default mode network" (DMN). The DMN is comprised of a set of brain regions exhibiting high metabolic activity during resting states (including sleep), and which also become deactivated during goal-directed cognition, in conjunction with upregulation of "task positive" brain areas [47]. Notably, DA signaling tends to shift activity in the direction of increased dominance by frontoparietal control networks, and 5-HT signaling tends to shift activity towards exhibiting greater DMN power [48]. Key nodes of the DMN include medial prefrontal and parietal cortices [49], [50], as well as the temporoparietal junction [51], [52], which together constitute key areas for imaginative simulations involving both self and other [53], [54], and which may also be essential for establishing minimum embodied selfhood and coherent subjective experience [13], [55]. These nodes become functionally and structurally connected in a gradual manner over the course of development [56], and may have been uniquely expanded in the course of human evolution [57]. With respect to capacities for imaginative planning, it is particularly notable that the DMN plays a central role in counterfactual mental simulations [58], [59]. The DMN is often considered to be at the top of the cortical hierarchy in FEP-AI [11], although evidence suggests a more complicated picture in which salience networks may be understood as constituting the highest levels of control [60]. Notably, 5-HT2a receptors are also particularly 
concentrated in the anterior insula [61], a key node in networks for salience determination and goal prioritization [62].

5-HT2a receptors are also responsible for the neuropsychological effects of psychedelics, which have been shown to elevate the entropy of endogenous brain activity and potentially enhance the richness of both the level and contents of consciousness [63]-[67]. With the widely known "RElaxed Beliefs Under pSychedelics" (REBUS) model [68], 5-HT2a agonists are suggested to attenuate the precision of high-level prior beliefs, so flattening the curvature of free energy landscapes and enhancing sensitivity to novel observations [69]. This altered regime promotes the breakdown of the brain's usual hierarchical structure [70], so allowing for an "anarchic" state in which novel forms of communication are allowed between brain areas, so allowing for enhanced cognitive exploration and opportunities for updating of deep beliefs. In this REBUS regime, ascending prediction errors from hierarchically lower levels may reshape upper level (potentially excessively precise) priors, so allowing the agent to break free from overly rigid patterns of thought and behavior.

In machine learning terms, such maladaptive cognitive and behavioral habits could be viewed as constituting self-adversarial policies, potentially formed through histories of excessive (or premature) exploitation in policy selection [71]. The more entropic dynamics afforded by high levels of 5-HT2a agonism, however, may allow agents to escape from these self-undermining attractors and reach more desirable regions of policy space. In terms of active inference, such relaxation of deep beliefs would be understood as reducing dominance from the parameters that serve as priors for agentbased generative models [72], which if excessively concentrated may preclude patterns of policy selection that could allow for opportunities for updating [73]. Concentration of probability mass in Dirichlet parameters via iterative policy selection and learning may provide a model of personality formation, and so their potential updating under conditions of strong 5-HT2a agonism (or functional homologues) could also provide a model of the kinds of personality change that have been associated with psychedelics [74]. This would also provide a model for the generation of novel, and potentially more (and possibly excessive) creative modes of cognition for both biological and artificial agents [75].

However, more physiologically typical low-to-moderate levels of 5-HT2a agonism have been suggested to involve a strengthening of beliefs under psychedelics (i.e., SEBUS effects) [76], both on account of increased activity from deep pyramidal neurons encoding prior expectations (or predictions), as well as reduced activity from superficial pyramidal neurons encoding prediction errors. Under this kind of SEBUS regime, individuals may engage in counterfactual processing under conditions ofintense salience (e.g. sophisticated inference with high affective charge), potentially including greater confidence in both imagination and action. This would be highly consistent with accounts of 5-HT2a signaling as entailing strategies for "active coping"in the face of uncertainty/threat [6]. Whether patterns of either simulated or overtly enacted policy selection are more exploitative or exploratory would depend on a multitude of both preexisting and context-specific priors over preferred patterns of enaction (e.g. typical levels of curious engagement) [77]. Further, both 5-HT1a and 5-HT2a signaling have been shown to inhibit sharp-wave ripples [78], which may 
correspond to hippocampal/entorhinal remapping events [79]. Such inhibition of resetting of (generalized) mapping and accompanying repertoires of operative policies may promote opportunities for imaginative planning via more extended rollouts [80]. However, while 5-HT1a signaling will tend to be associated with more passive forms of cognition and behavior as described above, 5-HT2a signaling could promote more proactive modes of engagement with elevated affect from enhanced sensitivity to interoceptive signals [36]. In this way, 5-HT2a would provide a flexible parameter for imaginative planning when systems face varying degrees of stress (or uncertainty) with respect to achieving their goals.

SEBUS effects may also occur alongside REBUS effects at moderate-to-high levels of 5-HT2a agonism [68], [76], with potentially further indirect strengthening of intermediate level beliefs associated with the perceptual synthesis underlying conscious experience. This conjunction of high levels of perceptual vividness with exploration of novel forms of cognition could provide the greatest opportunities for updating, which may be a crucially important intervention for systems suffering from self-adversarial modes of policy selection. That is, while more physiological levels of 5-HT2a signaling may afford more flexible and adaptive refinement of normal policies, very high levels of agonism may constitute a qualitatively different regime that could allow both biological and artificial systems to "change their mind" in profound ways capable of altering their overall character [81]. Whether such changes are beneficial or harmful to system performance will depend on a multitude of factors, with the "set and setting" of such interventions being of crucial importance for shaping the direction of future system evolution. Going forward, we are currently planning simulation experiments inwhich we will demonstrate how these principles may apply to (artificial) world- modeling active inferential agents.

\section{Acknowledgements}

We gratefully acknowledge partial funding support from the Waterloo-Huawei Joint Innovation Lab within the project "the Active Inferential Meta-Learning Engine".

\section{References}

[1] N. M. Barnes et al., "International Union of Basic and Clinical Pharmacology. CX. Classification of Receptors for 5-hydroxytryptamine; Pharmacology and Function," Pharmacol. Rev., vol. 73, no. 1, pp. 310-520, Jan. 2021, doi: 10.1124/pr.118.015552.

[2] I. Moutkine, E. L. Collins, C. Béchade, and L. Maroteaux, "Evolutionary considerations on 5-HT2 receptors," Pharmacol. Res., vol. 140, pp. 14-20, Feb. 2019, doi: 10.1016/j.phrs.2018.09.014.

[3] G. Zhang and R. W. Stackman, "The role of serotonin 5-HT2A receptors in memory and cognition," Front. Pharmacol., vol. 6, p. 225, 2015, doi: 10.3389/fphar.2015.00225.

[4] M. W. Johnson, P. S. Hendricks, F. S. Barrett, and R. R. Griffiths, "Classic psychedelics: An integrative review of epidemiology, therapeutics, mystical experience, and brain network function," Pharmacol. Ther., vol. 197, pp. 83102, May 2019, doi: 10.1016/j.pharmthera.2018.11.010. 
[5] R. Kraehenmann, "Dreams and Psychedelics: Neurophenomenological Comparison and Therapeutic Implications," Curr. Neuropharmacol., vol. 15, no. 7, pp. 1032-1042, 2017, doi: 10.2174/1573413713666170619092629.

[6] R. Carhart-Harris and D. Nutt, "Serotonin and brain function: a tale of two receptors," J. Psychopharmacol. Oxf. Engl., vol. 31, no. 9, pp. 1091-1120, Sep. 2017, doi: 10.1177/0269881117725915.

[7] L.-X. Shao et al., "Psilocybin induces rapid and persistent growth of dendritic spines in frontal cortex in vivo," Neuron, vol. 0, no. 0, Jul. 2021, doi: 10.1016/j.neuron.2021.06.008.

[8] K. J. Friston, T. FitzGerald, F. Rigoli, P. Schwartenbeck, and G. Pezzulo, “Active Inference: A Process Theory,” Neural Comput., vol. 29, no. 1, pp. 1-49, Jan. 2017, doi: 10.1162/NECO_a_00912.

[9] K. J. Friston, "The free-energy principle: a unified brain theory?," Nat. Rev. Neurosci., vol. 11, no. 2, pp. 127-138, Feb. 2010, doi: 10.1038/nrn2787.

[10] K. J. Friston, R. Rosch, T. Parr, C. Price, and H. Bowman, "Deep temporal models and active inference," Neurosci. Biobehav. Rev., vol. 77, pp. 388-402, 2017, doi: 10.1016/j.neubiorev.2017.04.009.

[11] R. L. Carhart-Harris and K. J. Friston, "The default-mode, ego-functions and free-energy: a neurobiological account of Freudian ideas," Brain J. Neurol., vol. 133, no. Pt 4, pp. 1265-1283, Apr. 2010, doi: 10.1093/brain/awq010.

[12] A. Safron, "The Radically Embodied Conscious Cybernetic Bayesian Brain: From Free Energy to Free Will and Back Again," Entropy, vol. 23, no. 6, Art. no. 6, Jun. 2021, doi: 10.3390/e23060783.

[13] A. Safron, “An Integrated World Modeling Theory (IWMT) of Consciousness: Combining Integrated Information and Global Neuronal Workspace Theories With the Free Energy Principle and Active Inference Framework; Toward Solving the Hard Problem and Characterizing Agentic Causation," Front. Artif. Intell., vol. 3, 2020, doi: 10.3389/frai.2020.00030.

[14] K. Friston, L. Da Costa, D. Hafner, C. Hesp, and T. Parr, "Sophisticated Inference," Jun. 2020, Accessed: Jun. 18, 2020. [Online]. Available: https://arxiv.org/abs/2006.04120v1

[15] C. Hesp, A. Tschantz, B. Millidge, M. Ramstead, K. Friston, and R. Smith, "Sophisticated Affective Inference: Simulating Anticipatory Affective Dynamics of Imagining Future Events," in Active Inference, Cham, 2020, pp. 179-186. doi: 10.1007/978-3-030-64919-7_18.

[16] H. C. Barron, R. Auksztulewicz, and K. Friston, "Prediction and memory: a predictive coding account," Prog. Neurobiol., p. 101821, May 2020, doi: 10.1016/j.pneurobio.2020.101821.

[17] O. Çatal, T. Verbelen, T. Van de Maele, B. Dhoedt, and A. Safron, "Robot navigation as hierarchical active inference," Neural Netw., vol. 142, pp. $192-$ 204, Oct. 2021, doi: 10.1016/j.neunet.2021.05.010.

[18] A. Johnston, C. J. McBain, and A. Fisahn, "5-Hydroxytryptamine1A receptoractivation hyperpolarizes pyramidal cells and suppresses hippocampal gamma oscillations via Kir3 channel activation," J. Physiol., vol. 592, no. 19, pp. 41874199, Oct. 2014, doi: 10.1113/jphysiol.2014.279083. 
[19] F. Mannella, K. Gurney, and G. Baldassarre, "The nucleus accumbens as a nexus between values and goals in goal-directed behavior: a review and a new hypothesis," Front. Behav. Neurosci., vol. 7, p. 135, 2013, doi: 10.3389/fnbeh.2013.00135.

[20] T. H. B. FitzGerald, R. J. Dolan, and K. J. Friston, "Dopamine, reward learning, and active inference," Front. Comput. Neurosci., vol. 9, Nov. 2015, doi: 10.3389/fncom.2015.00136.

[21] K. J. Friston, P. Schwartenbeck, T. FitzGerald, M. Moutoussis, T. Behrens, and R. J. Dolan, "The anatomy of choice: dopamine and decision-making," Philos. Trans. R. Soc. B Biol. Sci., vol. 369, no. 1655, Nov. 2014, doi: 10.1098/rstb.2013.0481.

[22] R. J. Moran et al., "The Protective Action Encoding of Serotonin Transients in the Human Brain," Neuropsychopharmacology, vol. 43, no. 6, Art. no. 6, May 2018, doi: 10.1038/npp.2017.304.

[23] C. D. Grossman, B. A. Bari, and J. Y. Cohen, "Serotonin neurons modulate learning rate through uncertainty," bioRxiv, p. 2020.10.24.353508, Oct. 2020, doi: 10.1101/2020.10.24.353508.

[24] Y. Ohmura et al., "Disruption of model-based decision making by silencing of serotonin neurons in the dorsal raphe nucleus," Curr. Biol., vol. 31, no. 11, pp. 2446-2454.e5, Jun. 2021, doi: 10.1016/j.cub.2021.03.048.

[25] Y.-L. Boureau and P. Dayan, "Opponency Revisited: Competition and Cooperation Between Dopamine and Serotonin," Neuropsychopharmacology, vol. 36, no. 1, Art. no. 1, Jan. 2011, doi: 10.1038/npp.2010.151.

[26] N. D. Daw, S. Kakade, and P. Dayan, "Opponent interactions between serotonin and dopamine," Neural Netw. Off. J. Int. Neural Netw. Soc., vol. 15, no. 4-6, pp. 603-616, Jul. 2002, doi: 10.1016/s0893-6080(02)00052-7.

[27] K. Doya, K. W. Miyazaki, and K. Miyazaki, "Serotonergic modulation of cognitive computations," Curr. Opin. Behav. Sci., vol. 38, pp. 116-123, Apr. 2021, doi: 10.1016/j.cobeha.2021.02.003.

[28] E. S. Bromberg-Martin, O. Hikosaka, and K. Nakamura, "Coding of task reward value in the dorsal raphe nucleus," J. Neurosci. Off. J. Soc. Neurosci., vol. 30, no. 18, pp. 6262-6272, May 2010, doi: 10.1523/JNEUROSCI.0015-10.2010.

[29] S. Yagishita, "Transient and sustained effects of dopamine and serotonin signaling in motivation-related behavior," Psychiatry Clin. Neurosci., vol. 74, no. 2, pp. 91-98, 2020, doi: 10.1111/pcn.12942.

[30] S. C. Hayes, A Liberated Mind: How to Pivot Toward What Matters. Penguin, 2019.

[31] S. Atasoy, G. Deco, and M. L. Kringelbach, "Playing at the Edge of Criticality: Expanded Whole-Brain Repertoire of Connectome-Harmonics," in The Functional Role of Critical Dynamics in Neural Systems, N. Tomen, J. M. Herrmann, and U. Ernst, Eds. Cham: Springer International Publishing, 2019, pp. 27-45. doi: 10.1007/978-3-030-20965-0_2.

[32] A. K. Davis, F. S. Barrett, and R. R. Griffiths, "Psychological flexibility mediates the relations between acute psychedelic effects and subjective decreases in 
depression and anxiety," J. Context. Behav. Sci., vol. 15, pp. 39-45, Jan. 2020, doi: 10.1016/j.jcbs.2019.11.004.

[33] R. T. Gerraty, J. Y. Davidow, K. Foerde, A. Galvan, D. S. Bassett, and D. Shohamy, "Dynamic flexibility in striatal-cortical circuits supports reinforcement learning," J. Neurosci., pp. 2084-17, Feb. 2018, doi: 10.1523/JNEUROSCI.2084-17.2018.

[34] D. Ha and J. Schmidhuber, "World Models," ArXiv180310122 Cs Stat, Mar. 2018, doi: 10.5281/zenodo.1207631.

[35] J.-P. Voigt and H. Fink, "Serotonin controlling feeding and satiety," Behav. Brain Res., vol. 277, pp. 14-31, Jan. 2015, doi: 10.1016/j.bbr.2014.08.065.

[36] O. R. Hjorth et al., "Expression and co-expression of serotonin and dopamine transporters in social anxiety disorder: a multitracer positron emission tomography study," Mol. Psychiatry, pp. 1-10, Dec. 2019, doi: 10.1038/s41380019-0618-7.

[37] A. Fotopoulou and M. Tsakiris, "Mentalizing homeostasis: the social origins of interoceptive inference-replies to Commentaries," Neuropsychoanalysis, vol. 19, no. 1, pp. 71-76, 2017.

[38] A. Ciaunica, A. Constant, H. Preissl, and A. Fotopoulou, "The First Prior: from Co-Embodiment to Co-Homeostasis in Early Life.” PsyArXiv, Jan. 05, 2021. doi: 10.31234/osf.io/twubr.

[39] I. V. Pavlova and M. P. Rysakova, "Effects of Administration of Serotonin 5HT1A Receptor Ligands into the Amygdala on the Behavior of Rats with Different Manifestations of Conditioned Reflex Fear," Neurosci. Behav. Physiol., vol. 48, no. 3, pp. 267-278, Mar. 2018, doi: 10.1007/s11055-018-05601.

[40] P. Dayan and Q. J. M. Huys, "Serotonin in Affective Control," Annu. Rev. Neurosci., vol. 32, no. 1, pp. 95-126, 2009, doi: 10.1146/annurev.neuro.051508.135607.

[41] L. Colyn, E. Venzala, S. Marco, I. Perez-Otaño, and R. M. Tordera, "Chronic social defeat stress induces sustained synaptic structural changes in the prefrontal cortex and amygdala," Behav. Brain Res., vol. 373, p. 112079, Nov. 2019, doi: 10.1016/j.bbr.2019.112079.

[42] P. B. Badcock, C. G. Davey, S. Whittle, N. B. Allen, and K. J. Friston, "The Depressed Brain: An Evolutionary Systems Theory," Trends Cogn. Sci., vol. 21, no. 3, pp. 182-194, Mar. 2017, doi: 10.1016/j.tics.2017.01.005.

[43] W. Schultz, "Neuronal Reward and Decision Signals: From Theories to Data," Physiol. Rev., vol. 95, no. 3, pp. 853-951, Jul. 2015, doi: 10.1152/physrev.00023.2014.

[44] J. W. Dalley and J. P. Roiser, "Dopamine, serotonin and impulsivity," Neuroscience, vol. 215, pp. 42-58, Jul. 2012, doi: 10.1016/j.neuroscience.2012.03.065.

[45] N. C. Di Pietro and J. K. Seamans, "Dopamine and serotonin interactions in the prefrontal cortex: insights on antipsychotic drugs and their mechanism of action," Pharmacopsychiatry, vol. 40 Suppl 1, pp. S27-33, Dec. 2007, doi: 10.1055/s2007-992133. 
[46] H. Lu and Q. Liu, "Serotonin in the Frontal Cortex: A Potential Therapeutic Target for Neurological Disorders," Biochem. Pharmacol. Open Access, vol. 6, no. 1, p. e184, Feb. 2017, doi: 10.4172/2167-0501.1000e184.

[47] E. Dohmatob, G. Dumas, and D. Bzdok, "Dark control: The default mode network as a reinforcement learning agent," Hum. Brain Mapp., vol. 41, no. 12, pp. 3318-3341, 2020, doi: 10.1002/hbm.25019.

[48] B. Conio et al., "Opposite effects of dopamine and serotonin on resting-state networks: review and implications for psychiatric disorders," Mol. Psychiatry, vol. 25, no. 1, pp. 82-93, Jan. 2020, doi: 10.1038/s41380-019-0406-4.

[49] P. Fransson and G. Marrelec, "The precuneus/posterior cingulate cortex plays a pivotal role in the default mode network: Evidence from a partial correlation network analysis," NeuroImage, vol. 42, no. 3, pp. 1178-1184, Sep. 2008, doi: 10.1016/j.neuroimage.2008.05.059.

[50] A. V. Utevsky, D. V. Smith, and S. A. Huettel, "Precuneus Is a Functional Core of the Default-Mode Network," J. Neurosci., vol. 34, no. 3, pp. 932-940, Jan. 2014, doi: 10.1523/JNEUROSCI.4227-13.2014.

[51] B. Baird, A. Castelnovo, O. Gosseries, and G. Tononi, "Frequent lucid dreaming associated with increased functional connectivity between frontopolar cortex and temporoparietal association areas," Sci. Rep., vol. 8, no. 1, p. 17798, Dec. 2018, doi: 10.1038/s41598-018-36190-w.

[52] M. S. A. Graziano, "The temporoparietal junction and awareness," Neurosci. Conscious., vol. 2018, no. 1, Jan. 2018, doi: 10.1093/nc/niy005.

[53] D. Hassabis, R. N. Spreng, A. A. Rusu, C. A. Robbins, R. A. Mar, and D. L. Schacter, "Imagine All the People: How the Brain Creates and Uses Personality Models to Predict Behavior," Cereb. Cortex, vol. 24, no. 8, pp. 1979-1987, Aug. 2014, doi: 10.1093/cercor/bht042.

[54] A. Guterstam, B. J. Bio, A. I. Wilterson, and M. Graziano, "Temporo-parietal cortex involved in modeling one's own and others' attention," eLife, vol. 10, p. e63551, Feb. 2021, doi: 10.7554/eLife.63551.

[55] C. G. Davey and B. J. Harrison, "The brain's center of gravity: how the default mode network helps us to understand the self," World Psychiatry, vol. 17, no. 3, pp. 278-279, Oct. 2018, doi: 10.1002/wps.20553.

[56] F. Fan et al., "Development of the default-mode network during childhood and adolescence: A longitudinal resting-state fMRI study," NeuroImage, vol. 226, p. 117581, Feb. 2021, doi: 10.1016/j.neuroimage.2020.117581.

[57] R. L. Buckner and L. M. DiNicola, "The brain's default network: updated anatomy, physiology and evolving insights," Nat. Rev. Neurosci., vol. 20, no. 10, pp. 593-608, Oct. 2019, doi: 10.1038/s41583-019-0212-7.

[58] D. Hassabis and E. A. Maguire, "The construction system of the brain," Philos. Trans. R. Soc. Lond. B. Biol. Sci., vol. 364, no. 1521, pp. 1263-1271, May 2009, doi: 10.1098/rstb.2008.0296.

[59] L. Faul, P. L. St. Jacques, J. T. DeRosa, N. Parikh, and F. De Brigard, "Differential contribution of anterior and posterior midline regions during mental simulation of counterfactual and perspective shifts in autobiographical 
memories," NeuroImage, vol. 215, p. 116843, Jul. 2020, doi: 10.1016/j.neuroimage.2020.116843.

[60] Y. Zhou, K. J. Friston, P. Zeidman, J. Chen, S. Li, and A. Razi, "The Hierarchical Organization of the Default, Dorsal Attention and Salience Networks in Adolescents and Young Adults," Cereb. Cortex N. Y. NY, vol. 28, no. 2, pp. 726737, Feb. 2018, doi: 10.1093/cercor/bhx307.

[61] A. M. Santangelo et al., "Insula serotonin 2A receptor binding and gene expression contribute to serotonin transporter polymorphism anxious phenotype in primates," Proc. Natl. Acad. Sci., vol. 116, no. 29, pp. 14761-14768, Jul. 2019, doi: 10.1073/pnas.1902087116.

[62] A. R. Rueter, S. V. Abram, A. W. MacDonald, A. Rustichini, and C. G.DeYoung, "The goal priority network as a neural substrate of Conscientiousness," Hum. Brain Mapp., vol. 39, no. 9, pp. 3574-3585, Sep. 2018, doi: 10.1002/hbm.24195.

[63] L. Barnett, S. D. Muthukumaraswamy, R. L. Carhart-Harris, and A. K. Seth, "Decreased directed functional connectivity in the psychedelic state," NeuroImage, vol. 209, p. 116462, Apr. 2020, doi: 10.1016/j.neuroimage.2019.116462.

[64] M. M. Schartner, R. L. Carhart-Harris, A. B. Barrett, A. K. Seth, and S. D. Muthukumaraswamy, "Increased spontaneous MEG signal diversity for psychoactive doses of ketamine, LSD and psilocybin," Sci. Rep., vol. 7, p. 46421, Apr. 2017, doi: 10.1038/srep46421.

[65] J. Aru, M. Suzuki, R. Rutiku, M. E. Larkum, and T. Bachmann, "Coupling the State and Contents of Consciousness," Front. Syst. Neurosci., vol. 13, Aug. 2019, doi: 10.3389/fnsys.2019.00043.

[66] R. L. Carhart-Harris, "The entropic brain - revisited," Neuropharmacology, vol. 142, pp. 167-178, Nov. 2018, doi: 10.1016/j.neuropharm.2018.03.010.

[67] R. L. Carhart-Harris et al., "The entropic brain: a theory of conscious states informed by neuroimaging research with psychedelic drugs," Front. Hum. Neurosci., vol. 8, p. 20, 2014.

[68] R. L. Carhart-Harris and K. J. Friston, "REBUS and the Anarchic Brain: Toward a Unified Model of the Brain Action of Psychedelics," Pharmacol. Rev., vol. 71, no. 3, pp. 316-344, Jul. 2019, doi: 10.1124/pr.118.017160.

[69] A. I. Luppi et al., "Connectome Harmonic Decomposition of Human Brain Dynamics Reveals a Landscape of Consciousness," bioRxiv, p. 2020.08.10.244459, Aug. 2020, doi: 10.1101/2020.08.10.244459.

[70] A. I. Luppi, R. L. Carhart-Harris, L. Roseman, I. Pappas, D. K. Menon, and E. A. Stamatakis, "LSD alters dynamic integration and segregation in the human brain," NeuroImage, vol. 227, p. 117653, Feb. 2021, doi: 10.1016/j.neuroimage.2020.117653.

[71] K. O. Stanley and J. Lehman, Why Greatness Cannot Be Planned: The Myth of the Objective. Springer, 2015.

[72] A. Safron and C. G. DeYoung, "Chapter 18 - Integrating Cybernetic Big Five Theory with the free energy principle: A new strategy for modeling personalities as complex systems," in Measuring and Modeling Persons and Situations, D. 
Wood, S. J. Read, P. D. Harms, and A. Slaughter, Eds. Academic Press, 2021, pp. 617-649. doi: 10.1016/B978-0-12-819200-9.00010-7.

[73] A. Constant, C. Hesp, C. G. Davey, K. J. Friston, and P. B. Badcock, "Why Depressed Mood is Adaptive: A Numerical Proof of Principle for an Evolutionary Systems Theory of Depression," Comput. Psychiatry, vol. 5, no. 1, Art. no. 1, Jun. 2021, doi: 10.5334/cpsy.70.

[74] D. Erritzoe, J. Smith, P. M. Fisher, R. Carhart-Harris, V. G. Frokjaer, and G. M. Knudsen, "Recreational use of psychedelics is associated with elevated personality trait openness: Exploration of associations with brain serotonin markers," J. Psychopharmacol. Oxf. Engl., p. 269881119827891, Feb. 2019, doi: $10.1177 / 0269881119827891$.

[75] M. Girn, C. Mills, L. Roseman, R. L. Carhart-Harris, and K. Christoff, "Updating the dynamic framework of thought: Creativity and psychedelics," NeuroImage, vol. 213, p. 116726, Jun. 2020, doi: 10.1016/j.neuroimage.2020.116726.

[76] A. Safron, "Strengthened beliefs under psychedelics (SEBUS)? A Commentary on 'REBUS and the Anarchic Brain: Toward a Unified Model of the Brain Action of Psychedelics." PsyArXiv, Nov. 30, 2020. doi: 10.31234/osf.io/zqh4b.

[77] P. Schwartenbeck, J. Passecker, T. U. Hauser, T. H. FitzGerald, M. Kronbichler, and K. J. Friston, "Computational mechanisms of curiosity and goal-directed exploration," eLife, vol. 8, 10 2019, doi: 10.7554/eLife.41703.

[78] R. ul Haq et al., "Serotonin dependent masking of hippocampal sharp wave ripples," Neuropharmacology, vol. 101, pp. 188-203, Feb. 2016, doi: 10.1016/j.neuropharm.2015.09.026.

[79] P. Latuske, O. Kornienko, L. Kohler, and K. Allen, "Hippocampal Remapping and Its Entorhinal Origin," Front. Behav. Neurosci., vol. 11, 2018, doi: 10.3389/fnbeh.2017.00253.

[80] C. O'Callaghan, I. C. Walpola, and J. M. Shine, "Neuromodulation of the mindwandering brain state: the interaction between neuromodulatory tone, sharp wave-ripples and spontaneous thought," Philos. Trans. R. Soc. Lond. B. Biol. Sci., vol. 376, no. 1817, p. 20190699, Feb. 2021, doi: 10.1098/rstb.2019.0699.

[81] M. Pollan, How to Change Your Mind: The New Science of Psychedelics. Penguin Books Limited, 2018. 